03

\title{
О массопереносе, порожденном волновым возмущением поверхности тангенциального разрыва поля скоростей
}

\author{
(С) Д.Ф. Белоножко, А.А. Очиров \\ Ярославский государственный университет им. П.Г. Демидова, \\ 150000 Ярославль, Россия \\ e-mail: belonozhko@mail.ru
}

(Поступило в Редакцию 21 мая 2017 г.)

Построена аналитическая асимптотическая модель относительного массопереноса в системе двух идеальных несмешивающихся перемещающихся относительно друг друга жидкостей, по поверхности раздела которых распространяется капиллярно-гравитационная периодическая волна.

DOI: 10.21883/JTF.2018.05.45894.2315

\section{Введение}

Еще в XIX в. Г. Стокс сумел оценить скорость [1]

$$
V_{S}=a^{2} \omega k \exp (-2 k d)
$$

с которой происходит горизонтальный массоперенос, осуществляемый капиллярно-гравитационной волной, распространяющейся по свободной горизонтальной поверхности идеальной несжимаемой бесконечно глубокой жидкости. В стоксовской модели амплитуда волны $a$ считается малой по сравнению с ее длиной; параметр $k$ характеризует волновое число, $\omega-$ круговую частоту. Скорость дрейфа Стокса направлена в сторону распространения волны, пропорциональна квадрату ее амплитуды и экспоненциально убывает с глубиной $d$. Обычно распространение периодической волны с амплитудой $a$ связывают с вертикальными уменьшающимися с глубиной круговыми движениями материальных частиц среды [2-4] (для частичек, непосредственно прилегающих к поверхности, радиус витка примерно равен $a$ ). Но следует понимать, что из-за затухания движения с глубиной нижняя часть петли, описываемой материальной частичкой, оказывается меньше верхней. В результате через каждый период своего циклического движения частичка возвращается не в предыдущую верхнюю позицию, а в положение, несколько смещенное в направлении распространения волны [5]. С течением времени такие смещения складываются в средний дрейф, который неоднократно рассматривался в рамках самых различных моделей волнового массопереноса [6-10].

Между тем волновое движение на границе раздела двух сред - тема существенно более многоплановая, особенно если эти среды участвуют в относительном сдвиговом смещении $[11,12]$. Основное внимание здесь обычно уделяется неустойчивости границы раздела. В классическом случае говорят о неустойчивости Кельвина-Гельмгольца, условия реализации которой определяются из дисперсионного уравнения $[13,14]$ :

$$
\omega=\frac{k \rho^{\prime} U_{0} \pm \sqrt{k g\left(\rho^{2}-\rho^{\prime 2}\right)+k^{3} \mathcal{\gamma}\left(\rho+\rho^{\prime}\right)-k^{2} \rho \rho^{\prime} U_{0}^{2}}}{\rho+\rho^{\prime}}
$$

связывающего круговую частоту волнового движения $\omega$ с волновым числом $k$. Амплитуда волны здесь, так же как и в теории дрейфа Стокса, считается малой по сравнению с длиной, а сама волна распространяется по горизонтальной поверхности раздела двух идеальных несжимаемых несмешивающихся жидкостей, находящихся в поле тяжести g, ничем не ограниченных, кроме поверхности раздела. Считается, что плотность верхней жидкости меньше плотности нижней $\rho^{\prime}<\rho$, а величина коэффициента поверхностного натяжения границы раздела характеризуется параметром $\gamma$. При отсутствии волнового возмущения граница раздела горизонтальна, нижняя среда покоится, а верхняя (в силу идеальности модели жидкости), не испытывая трения, движется относительно нее со скоростью $U_{0}$ (скорость $U_{0}$ существенно дозвуковая, чтобы обе жидкости можно было считать несжимаемыми [15]). Если $U_{0}$ недостаточно велика для отрицательности подкоренного выражения, входящего в (2), то любая простейшая периодическая волна распространяется по поверхности раздела без изменения своей амплитуды. Если же величина $U_{0}$ оказывается достаточно большой, чтобы появился интервал значений $k$, при которых под корнем возникает отрицательное число, амплитуда соответствующих волновых возмущений, пусть даже изначально незначительных, начинает экспоненциально расти во времени, инициируя начало развития неустойчивости Кельвина-Гельмгольца [16,17].

В своем классическом виде теория волнового движения на горизонтальной поверхности тангенциального разрыва поля скоростей оставляет без внимания вопросы взаимосвязи волнового движения с возникающим при этом относительным горизонтальным массопереносом жидкостей. Настоящая работа нацелена на заполнение этого пробела: в рамках классической модели течения, отвечающего дисперсионному уравнению (2), бу- 
дут установлены принципы расчета траекторий жидких частиц, выведены простые аналитические соотношения, аналогичные выражению (1), для оценки интенсивности волнового массопереноса.

\section{1. Формулировка задачи}

Две идеальные несжимаемые несмешивающиеся жидкости, находясь в поле тяжести g, заполняют в декартовой прямоугольной системе координат Oxyz c осью $O z$, направленной вертикально вверх, полупространства $z>0$ и $z<0$. Верхняя среда с плотностью $\rho^{\prime}$ поступательно движется вдоль горизонтальной оси $O x$ со скоростью $U_{0}$ относительно нижней более тяжелой, обладающей плотностью $\rho>\rho^{\prime}$. Рассматривается периодическое волновое возмущение $z=\xi(x, t)$ границы раздела с волновым числом $k$, распространяющееся вдоль оси $O x$. Течение для простоты считается независящим от второй горизонтальной координаты $y$. Коэффициент поверхностного натяжения на границе раздела характеризуется параметром $\gamma$. Задача состоит в установлении характера перемещения материальных частиц обеих сред в зависимости от значения скорости $U_{0}$, амплитуды и длины волнового возмущения.

Может показаться, что задачу следует сразу формулировать в переменных Лагранжа [13], в которых вектор $\mathbf{V}_{L}\left(\mathbf{r}_{0}, t\right)$ и скаляр $p_{L}\left(\mathbf{r}_{0}, t\right)$ описывают скорость малой материальной частички и давление в позиции ее текущего положения, а радиус-вектор $\mathbf{r}_{0}$ отмечает начальное положение этой частицы в момент времени $t=0$. Но важно понимать, что в случае идеальных жидкостей с общей границей лагранжев подход плохо приспособлен к работе с условиями на поверхности их раздела. Действительно, пусть $\mathbf{r}_{0}-$ положение на границе раздела, к которому при $t=0$,прилегают“ c разных сторон две малые материальные частички, относящиеся к разным средам. Через конечное время $t$ обе частички, совершив в общем случае разные (что допустимо на границе идеальных жидкостей) тангенциальные перемещения, окажутся в разных точках этой поверхности, а значит, в лагранжевых координатах функции давления $p_{L}\left(\mathbf{r}_{0}, t\right)$ и $p_{L}^{\prime}\left(\mathbf{r}_{0}, t\right)$ в произвольный момент времени окажутся сопоставленными разным точкам границы. Ясно, что условие баланса давления вдоль поверхности совершенно недопустимо записывать в виде $p_{L}\left(\mathbf{r}_{0}, t\right)=p_{L}^{\prime}\left(\mathbf{r}_{0}, t\right)$. Корректная же форма записи этого условия в рамках лагранжевого описания представляется далеко неочевидной.

В связи со сказанным, задачу целесообразнее формулировать и решать в переменных Эйлера, в которых скорость течения $\mathbf{V}(\mathbf{r}, t)$ связана с пространственной точкой $\mathbf{r}$ в текущий момент времени $t$, через которую, сменяя друг друга, проходят разные жидкие частички. При этом скорость материальных частиц (переход к лагранжевому представлению) придется определять с помощью дополнительных соображений.
Движение идеальных жидкостей будем считать потенциальным с потенциалами скоростей $\varphi$ и $\varphi^{\prime}$ в нижней и верхней областях: $\mathbf{V}(\mathbf{r}, t)=\nabla \varphi, \mathbf{V}^{\prime}(\mathbf{r}, t)=U_{0} \mathbf{e}_{x}+\nabla \varphi^{\prime}$. Потенциал $\varphi^{\prime}$ определяет малую добавку к постоянной компоненте поля скоростей $U_{0} \mathbf{e}_{x}$ в верхней области $\left(\mathbf{e}_{x}-\right.$ направляющий орт $\left.O x\right)$. Задача определения потенциалов $\varphi$ и $\varphi^{\prime}$ имеет вид:

$$
\begin{gathered}
z>\xi: \Delta \varphi^{\prime}=0 \\
P^{\prime}=p_{0}-\rho^{\prime} g z-\rho^{\prime} \partial_{t} \varphi^{\prime}-\left(\rho^{\prime} / 2\right)\left[\left(\partial_{x} \varphi^{\prime}+U_{0}\right)^{2}\left(\partial_{z} \varphi^{\prime}\right)^{2}\right] \\
\partial_{t} \xi+\partial_{x} \xi \partial_{x} \varphi=\partial_{z} \varphi ; \quad \partial_{t} \xi+\left(\partial_{x} \varphi^{\prime}+U_{0}\right) \partial_{x} \xi=\partial_{z} \varphi^{\prime} \\
P-P^{\prime}=-\gamma \partial_{x x} \xi\left(1+\left(\partial_{x} \xi\right)^{2}\right)^{-3 / 2} \\
z<\xi: \quad \Delta \varphi=0 ; \\
P=p_{0}-\rho g z-\rho \partial_{t} \varphi-(\rho / 2)\left[\left(\partial_{x} \varphi\right)^{2}+\left(\partial_{z} \varphi\right)^{2}\right] \\
z \rightarrow \infty: \nabla \varphi^{\prime} \rightarrow 0 ; \quad z \rightarrow-\infty: \nabla \varphi \rightarrow 0
\end{gathered}
$$

Здесь $p_{0}$ - постоянное внешнее давление, которое в случае сред вода-воздух равно атмосферному; $P$ и $P^{\prime}-$ гидродинамические давления в нижней и верхней средах.

Задача решалась методом разложения по малому параметру $\varepsilon=k a$, пропорциональному отношению амплитуды волны $a$ к ее длине $\lambda=2 \pi / k$. В дальнейшем для краткости, подразумевая малость по $\varepsilon$, будем просто говорить о порядке малости по амплитуде волны. Поскольку уже в простейшем случае, рассмотренном Стоксом, дрейфовые компоненты течения имеют второй порядок малости по амплитуде волны, целесообразно рассмотреть разложение до второго порядка малости:

$$
\begin{gathered}
\varphi=\varphi_{1}+\varphi_{2}+O\left(\varepsilon^{3}\right) ; \quad \varphi^{\prime}=\varphi_{1}^{\prime}+\varphi_{2}^{\prime}+O\left(\varepsilon^{3}\right) ; \\
\xi=\xi_{1}+\xi_{2}+O\left(\varepsilon^{3}\right) .
\end{gathered}
$$

Здесь $O$ - символ порядка: $\varphi_{q}=O\left(\varepsilon^{q}\right), \varphi_{q}^{\prime}=O\left(\varepsilon^{q}\right)$, $\xi_{q}=O\left(\varepsilon^{q}\right)$.

Выражая неизвестные функции и их производные, определенные на границе $z=\xi(x, t)$ через степенное разложение по $\xi$ в окрестности $z=0$, граничные условия (4) несложно свести к условиям на уровне $z=0$, после чего с помощью (7) разбить (3)-(6) на задачи разных порядков малости.

Задача первого по $\varepsilon$ порядка малости имеет вид

$$
\begin{gathered}
z>0: \Delta \varphi_{1}^{\prime}=0 ; \quad z<0: \Delta \varphi_{1}=0 ; \\
z=0: \quad \partial_{t} \xi_{1}-\partial_{z} \varphi_{1}=0 ; \partial_{t} \xi_{1}+U_{0} \partial_{x} \xi_{1}-\partial_{z} \varphi_{1}^{\prime}=0 ; \\
g \xi_{1}\left(\rho^{\prime}-\rho\right)-\rho \partial_{t} \varphi_{1}+\rho^{\prime} \partial_{t} \varphi_{1}^{\prime}+\rho^{\prime} U_{0} \partial_{x} \varphi_{1}^{\prime}+\gamma \partial_{x} \xi_{1}=0 ; \\
z \rightarrow \infty: \nabla \varphi_{1}^{\prime} \rightarrow 0 ; \quad z \rightarrow-\infty: \nabla \varphi_{1} \rightarrow 0 .
\end{gathered}
$$

Задачи второго порядка малости задаются соотношениями:

$$
\begin{aligned}
& z>0: \Delta \varphi_{2}^{\prime}=0 ; \quad z<0: \Delta \varphi_{2}=0 \\
& z=0: \quad \partial_{t} \xi_{2}-\partial_{z} \varphi_{2}=\xi_{1} \partial_{z z} \varphi_{1}-\partial_{x} \varphi_{1} \xi_{1}
\end{aligned}
$$




$$
\begin{gathered}
\partial_{t} \xi_{2}+U_{0} \partial_{x x} \xi_{2}-\partial_{z} \varphi_{2}^{\prime}=\xi_{1} \partial_{z z} \varphi_{1}^{\prime}-\partial_{x x} \varphi_{1}^{\prime} \partial_{x x} \xi_{1} ; \\
g \xi_{2}\left(\rho^{\prime}-\rho\right)-\rho \partial_{t} \varphi_{2}+\rho^{\prime} \partial_{t} \varphi_{2}^{\prime}+\rho^{\prime} U_{0} \partial_{x} \varphi_{2}^{\prime}+\gamma \partial_{x x} \xi_{2} \\
=\rho \xi_{1} \partial_{z t} \varphi_{1}+(\rho / 2)\left(\left(\partial_{x} \varphi_{1}\right)^{2}+\left(\partial_{z} \varphi_{1}\right)^{2}\right)-\rho^{\prime} \xi_{1} \partial_{z t} \varphi_{1}^{\prime} \\
-\left(\rho^{\prime} / 2\right)\left(\left(\partial_{x} \varphi_{1}^{\prime}\right)^{2}+\left(\partial_{z} \varphi_{1}^{\prime}\right)^{2}+2 U_{0} \xi_{1} \partial_{x x} \varphi_{1}^{\prime}\right) ; \\
z \rightarrow \infty: \nabla \varphi_{2}^{\prime} \rightarrow 0 ; \quad z \rightarrow-\infty: \nabla \varphi_{2} \rightarrow 0 .
\end{gathered}
$$

Как и в большинстве классических аналитических построений периодических волновых профилей на поверхности идеальных жидкостей, вместо начального условия использовалась концепция поиска решения, которое в аналитическом плане наиболее просто описывает периодическую бегущую волну с волновым числом $k$.

\section{2. Свойства решения}

В первом приближении по амплитуде волны простейшее волновое решение задачи имеет вид

$$
\begin{gathered}
\xi_{1}=\frac{1}{2} a \exp (i(\omega t-k x))+\text { c.c.; } \\
\varphi_{1}^{\prime}=-\frac{1}{2} \frac{i}{k}\left(\omega-k U_{0}\right) a \exp (i(\omega t-k x)) \exp (-k z)+\text { c.c.; } \\
\varphi_{1}=\frac{1}{2} \frac{i \omega}{k} a \exp (i(\omega t-k x)) \exp (k z)+\text { c.c. }
\end{gathered}
$$

Здесь $i-$ мнимая единица, а „с.с.““ означает комплексно сопряженные слагаемые. Частота $\omega$ удовлетворяет дисперсионному уравнению (2).

Из (11), (12) легко выразить эйлерово поле скоростей в первом приближении по амплитуде волны. Для нижней среды горизонтальная $u_{1}$ и вертикальная $v_{1}$ компоненты скорости описываются выражениями

$$
\begin{aligned}
& u_{1}=\frac{1}{2} a \omega \exp (i(\omega t-k x)) \exp (k z)+\text { c.c.; } \\
& v_{1}=-\frac{i}{2} a \omega \exp (i(\omega t-k x)) \exp (k z)+\text { c.c. }
\end{aligned}
$$

В верхней области, включая в выражение для горизонтальной скорости постоянную составляющую $U_{0} \mathbf{e}_{x}$, имеем соотношения

$$
\begin{aligned}
& u_{1}^{\prime}=U_{0}-\frac{1}{2} a\left(\omega-k U_{0}\right) \exp (i(\omega t-k x)) \exp (-k z)+\text { c.c.; } \\
& v_{1}^{\prime}=\frac{i}{2} a\left(\omega-k U_{0}\right) \exp (i(\omega t-k x)) \exp (-k z)+\text { c.c. }
\end{aligned}
$$

Из дисперсионного уравнения (2) следует, что если

$$
U_{0}^{2} \leq U_{\mathrm{cr}}^{2}(k)=\frac{k^{2} \gamma\left(\rho+\rho^{\prime}\right)+g\left(\rho^{2}-\rho^{\prime 2}\right)}{k \rho \rho^{\prime}},
$$

то частота $\omega$ будет действительным числом и, значит, компоненты скорости обеих жидкостей являются периодическими функциями $\propto \cos (\omega t-k x)$ или $\propto \sin (\omega t-k x)$. Если же $U_{0}^{2}>U_{\mathrm{cr}}^{2}(k)$, то частота $\omega$ становится комплексной, и компоненты скорости оказываются пропорциональны $\propto \exp ( \pm \operatorname{Im}(\omega) t) \cos (\operatorname{Re}(\omega) t-k x)$ или $\propto \exp ( \pm \operatorname{Im}(\omega) t) \sin (\operatorname{Re}(\omega) t-k x)$, что соответствует волновым движениям с экспоненциально изменяющейся амплитудой. В обоих случаях, принимая во внимание наличие периодического множителя, будем говорить о циклическом характере выражений. При выполнении условия $U_{0}^{2}>U_{\mathrm{cr}}^{2}(k)$ одним из решений задачи является волновое возмущение с волновым числом $k$ и экспоненциально растущей амплитудой. Именно такие возмушения формируют начальную стадию развития неустойчивости Кельвина-Гельмгольца, о которой говорилось во введении.

Подставляя соотношения (10)-(12) в правые части граничных условий (8), (9) задачи второго порядка малости, несложно установить, что неоднородности граничных условий становятся циклическими выражениями $\propto \exp (2 i(\omega t-k x))$. Этим же свойством будет обладать и само решение задачи второго порядка малости. В дальнейшем принципиальную роль будет играть именно это свойство решения задачи второго порядка малости, а не конкретный вид этого решения.

\section{3. Переход к переменным Лагранжа}

Чтобы рассчитать движение материальных частичек, необходимо перейти к лагранжевому представлению поля скоростей. В условиях, когда причиной движения является периодическое малоаплитудное волновое возмущение неподвижной жидкости, лагранжева скорость $\mathbf{V}_{L}\left(\mathbf{r}_{0}, t\right)$ выражается через эйлерову $\mathbf{V}(\mathbf{r}, t)$, взятую в точке $\mathbf{r}=\mathbf{r}_{0}$, соответствующей начальному положению материальной частицы, по известной формуле $[14,18-21]$ :

$$
\mathbf{V}_{L}\left(\mathbf{r}_{0}, t\right)=\mathbf{V}\left(\mathbf{r}_{0}, t\right)+\left(\left(\int_{0}^{t} \mathbf{V}\left(\mathbf{r}_{0}, \tau\right) d \tau\right) \nabla_{0}\right) \mathbf{V}(\mathbf{r}, t)
$$

Индекс „““ при операторе Гамильтона $\nabla$ означает, что соответствующие производные должны вычисляться при $\mathbf{r}=\mathbf{r}_{0}-$ в начальном положении отслеживаемой материальной частички. В основе вывода формулы (16) лежит разложение эйлеровой скорости по малому смещению материальной частицы от положения $\mathbf{r}_{0}$ в момент времени $t$ [12]. Поэтому важным условием применимости (16) является малость удаления материальной частички от начального положения: за конечное время $t$ она не должна смещаться на расстояние, большее амплитуды волны. Сама формула (16) справедлива во втором приближении амплитуде волны.

В первом по амплитуде приближении применение (16) к полю скоростей (13) сводится к добавлению 
индекса „0“ к символам координат:

$$
\begin{aligned}
& u_{L 1}=\frac{1}{2} a \omega \exp \left(i\left(\omega t-k x_{0}\right)\right) \exp \left(k z_{0}\right)+\text { c.c.; } \\
& v_{L 1}=-\frac{i}{2} a \omega \exp \left(i\left(\omega t-k x_{0}\right)\right) \exp \left(k z_{0}\right)+\text { c.c. }
\end{aligned}
$$

Теперь $\left(x_{0}, z_{0}\right)$ это не точка, в которой определяется вектор скорости течения в текущий момент времени, а координаты начального положения материальной частички - изменился статус координатных переменных. Формальное совпадение выписанных выражений для лагражевой и эйлеровой скоростей в первом по амплитуде приближении хорошо известно и неоднократно использовалось для установления примерно круговой формы траекторий частиц, участвующих в волновом движении [2,3].

Прямое применение формулы (16) к вектору скорости верхней среды (14) недопустимо, поскольку ее частички в системе координат $O x y z$ участвуют в общем горизонтальном сдвиге со скоростью $U_{0}$ и за конечное время $t$ значительно смещаются от своего начального положения. Для исправления ситуации следует перейти в новую систему координат $O^{*} x^{*} y^{*} z^{*}$, которая совпадает с $O x y z$ при $t=0$ и с течением времени переносится со скоростью $U_{0}$ вдоль оси $O x$. Учитывая связь старой и новой горизонтальной координаты $x=x^{*}+U_{0} t$, несложно заметить, что с переходом в новую координатную систему в полном соответствии с эффектом Допплера изменяется частота волнового движения:

$$
\begin{gathered}
\omega t-k x=\omega t-k\left(x^{*}+U_{0} t\right)=\Omega t-k x^{*} ; \quad \Omega=\omega-k U_{0} \\
\cos (\omega t-k x) \rightarrow \cos \left(\Omega t-k x^{*}\right) \\
\sin (\omega t-k x) \rightarrow \sin \left(\Omega t-k x^{*}\right)
\end{gathered}
$$

Эйлерово поле скоростей в первом приближении по амплитуде волны в движущейся системе координат будет описываться соотношениями

$$
\begin{aligned}
& u_{1}^{\prime *}=-\frac{1}{2} a \Omega \exp \left(i\left(\Omega t-k x^{*}\right)\right) \exp \left(-k z^{*}\right)+\text { c.c.; } \\
& v_{1}^{\prime *}=\frac{i}{2} a \Omega \exp \left(i\left(\Omega t-k x^{*}\right)\right) \exp \left(-k z^{*}\right)+\text { c.c. }
\end{aligned}
$$

Теперь применение формулы (16) правомерно и в первом приближении по амплитуде волны снова сводится к приписыванию к координатным переменным индекса „0“, означающего изменение статуса координат на лагранжевые. Обратный переход в систему $O x y z$ дает искомые компоненты лагранжевой скорости в неподвижной системе:

$$
\begin{aligned}
& u_{L 1}^{\prime}=U_{0}-\frac{1}{2} a \Omega \exp \left(i\left(\Omega t-k x^{*}\right)\right) \exp \left(-k z_{0}\right)+\text { c.c.; } \\
& v_{L 1}^{\prime}=\frac{i}{2} a \Omega \exp \left(i\left(\Omega t-k x^{*}\right)\right) \exp \left(-k z_{0}\right)+\text { c.c. }
\end{aligned}
$$

В связи с совпадением $O^{*} x^{*} y^{*} z^{*}=O x y z$ при $t=0$ для лагражевых координат выполняются равенства $x_{0}=x_{0}^{*}, z_{0}=z_{0}^{*}$ и никаких преобразований, обратных к (18), влекущих изменение частоты $\Omega$, не возникает, поскольку теперь речь идет не о частоте волнового движения в распределенной системе, а о частоте циклических движений отдельной материальной частички. Здесь можно провести аналогию с частотой колебаний маятника: в рамках классической механики она одинакова во всех системах отсчета.

\section{4. Дрейфовые и циклические составляющие скорости}

Из (16) следует, что горизонтальная скорость частички нижней среды во втором приближении по амплитуде волны рассчитывается по формуле:

$$
\begin{aligned}
& u_{L}\left(x_{0}, z_{0}, t\right)=u_{1}\left(x_{0}, z_{0}, t\right)+u_{2}\left(x_{0}, z_{0}, t\right) \\
& +\left(\int_{0}^{t} u_{1}\left(x_{0}, z_{0}, \tau\right) d \tau\right)\left(\frac{\partial u_{1}(x, z, t)}{\partial x}\right) \\
& +\left(\int_{0}^{t} v_{1}\left(x_{0}, z_{0}, \tau\right) d \tau\right)\left(\frac{\partial u_{1}(x, z, t)}{\partial z}\right)_{0} .
\end{aligned}
$$

Для выделения из этого выражения дрейфовой компоненты следует заметить, что слагаемые $u_{1}\left(x_{0}, z_{0}, t\right)$ и $u_{2}\left(x_{0}, z_{0}, t\right)$ являются циклическими (см. (13) и заключительный тезис разд. 2), и поэтому никакого вклада в дрейф не вносят. Вычисление интегральных слагаемых с подстановкой в них соотношений (13) приводит к выражению, которое наряду с циклическими слагаемыми содержит искомую компоненту скорости горизонтального дрейфа в направлении оси $O x$ (в направлении распространения волны):

если

$$
U_{0}^{2} \leq U_{\mathrm{cr}}^{2}(k): \quad V_{S}=a^{2} \omega k \exp (2 k z)
$$

если

$$
U_{0}^{2}>U_{\mathrm{cr}}^{2}(k): \quad V_{S}=a^{2} \sigma k \exp (2 r t) \exp (2 k z) .
$$

При $U_{0}^{2}>U_{\mathrm{cr}}^{2}(k)$ (см. (15)) частота $\omega$, вычисленная с помощью дисперсионного уравнения (2), оказывается комплексным числом. Для ее действительной и мнимой частей использованы вспомогательные обозначения:

$$
\begin{gathered}
U_{0}^{2}>U_{\mathrm{cr}}^{2}(k): \quad \sigma=\operatorname{Re}(\omega) ; r=\operatorname{Im}(\omega) ; \\
\sigma=\frac{k \rho^{\prime} U_{0}}{\rho+\rho^{\prime}} ; \\
r=\frac{\sqrt{k^{2} \rho \rho^{\prime} U_{0}^{2}-k g\left(\rho^{2}-\rho^{\prime 2}\right)-k^{3} \gamma\left(\rho+\rho^{\prime}\right)}}{\rho+\rho^{\prime}} .
\end{gathered}
$$

Параметр $r$ положителен для одного корня дисперсионного уравнения и отрицателен для другого. Здесь и в 
дальнейшем интересно взять корень с $r>0$, характеризующий инкремент развития неустойчивости волнового возмушения с волновым числом $k$. Соотношение (21) с точностью до обозначений совпадает с выражением (1) для классического дрейфа Стокса (для сравнения с (1) нужно учесть, что в $(21) z<0)$.

Расчет скорости дрейфа в верхней среде осуществляется аналогично. Но перед применением формулы (16) по причинам, изложенным в предыдущем разделе, следует перейти в систему отсчета, движущуюся вместе с верхней средой, а потом вновь вернуться в неподвижную систему. При этом частота циклических выражений изменится по правилам, описанным в разд. 3. Несложно получить, что скорость дрейфового горизонтального переноса материальных частиц верхней среды в проекции на направление $O x$ вычисляется по формулам: если

$$
U_{0}^{2} \leq U_{\mathrm{cr}}^{2}(k): \quad V_{S}^{\prime}=U_{0}+a^{2}\left(\omega-k U_{0}\right) k \exp (-2 k z) ;
$$

если

$U_{0}^{2}>U_{\mathrm{cr}}^{2}(k): V_{S}^{\prime}=U_{0}+a^{2}\left(\sigma-k U_{0}\right) k \exp (2 r t) \exp (-2 k z)$.

Интересно обратить внимание на то, что, несмотря на свою малость, дрейф (21), через достаточно длительный по сравнению с периодом циклических движений материальных частиц промежуток времени, снесет частички нижней среды вдоль $O x$ на расстояние, значительно превышающее амплитуду волны. Формула (16) на таких временах перестает быть справедливой. Для полной корректности ее следовало бы применить к эйлеровой скорости в системе отсчета, движущейся вместе со средним дрейфом материальной частички, а потом перейти назад в неподвижную систему. Применяя эту идею к полю скоростей (13) и не забывая изменить частоту (см. предыдущий разд.) новым значением $\omega-k V_{S}$, несложно найти, что в нижней жидкости циклические компоненты скорости правильнее записывать в усовершенствованной форме:

$$
\begin{aligned}
& u_{L 1}=\frac{1}{2} a \omega \exp \left(i\left(\left(\omega-k V_{S}\right) t-k x_{0}\right)\right) \exp \left(k z_{0}\right)+\text { c.c.; } \\
& v_{L 1}=-\frac{i}{2} a \omega \exp \left(i\left(\left(\omega-k V_{S}\right) t-k x_{0}\right)\right) \exp \left(k z_{0}\right)+\text { c.c. }
\end{aligned}
$$

Согласно (26), период $\tau=2 \pi /\left(\omega-k V_{S}\right)$ циклического движения материальной частицы больше периода $T=2 \pi / \omega$ волнового движения границы раздела (10). Этот факт принципиален в вопросе согласования движения границы раздела (10) как геометрического места точек, разделяющих среды, с движением материальных частиц, прилегающих к этой поверхности и участвующих как в циклической циркуляции (26), так и в дрейфовом переносе (21).

Действительно, пусть положение материальной частички в начальный момент времени $t_{0}$ характеризуется горизонтальной координатой $x_{0}$, и частичка располагается на вершине одного из горбов (назовем его первым) периодической волны (10), распространяющейся в направлении оси $O x$. Через период своего циклического движения $\tau$ эта частичка должна снова оказаться в верхнем положении. За время $\tau$ она совершает петлеобразное движение: сначала „ныряет“ вниз вдоль $O x$; затем по нижней части витка перемещается против $O x$; переходит на верхнюю дугу петли и снова двигается вдоль $O x$, возвращаясь в свое самое верхнее положение в тот момент, когда в эту же точку прибывает вершина второго горба, следовавшего за первым. Поскольку время $\tau$ больше периода волнового движения $T$, вершина второго горба в момент времени $t=t_{0}+\tau$ окажется не над координатой $x_{0}$, а в положении $x=x_{0}+V_{\mathrm{ph}}(\tau-T)$, где $V_{\mathrm{ph}}=\omega / k-$ фазовая скорость волны (10). Таким образом, за период собственного циклического движения $\tau$ материальная частичка сместится по горизонтали на расстояние $\Delta x=\omega(\tau-T) / k$. Разделив $\Delta x$ на $\tau$, получим скорость систематического смещения материальной частицы вдоль $O x$. Из (10) и (26) следует, что периоды рассматриваемых типов движений: $T=2 \pi / \omega$ и $\tau=2 \pi /\left(\omega-k V_{S}\right)$. Несложно убедиться, что

$\frac{\Delta x}{\tau}=\frac{(\tau-T) \omega}{k \tau}=\left(\frac{2 \pi}{\omega-k V_{S}}-\frac{2 \pi}{\omega}\right) \frac{\omega}{k} \frac{\left(\omega-k V_{S}\right)}{2 \pi}=V_{S}$.

Таким образом, именно при указанных значениях $\tau$ и $T$ циклическое движение (26) частиц среды и волновое движение границы раздела (10) оказываются согласованными с дрейфовым перемещением материальных частиц нижней среды (21). Если не учитывать разницу между частотой циклического движения самой частицы и частотой волнового движения распределенной системы частиц, модель получится рассогласованной: совершающая витки и перемещающаяся вдоль горизонтали материальная частичка не будет с течением времени оставаться на смещающемся с фазовой скоростью профиле волнового искажения.

Аналогичные рассуждения вносят поправку в формулы (19) для скорости циклического движения материальных частиц верхней среды

$$
\begin{aligned}
& u_{L 1}^{\prime}=U_{0}-\frac{1}{2} a \Omega \exp \left(i\left(\Omega-k V_{S}^{\prime}\right) t-k x_{0}\right) \exp \left(-k z_{0}\right)+\text { c.c.; } \\
& v_{L 1}^{\prime}=\frac{i}{2} a \Omega \exp \left(i\left(\Omega-k V_{S}^{\prime}\right) t-k x_{0}\right) \exp \left(-k z_{0}\right)+\text { c.c. }
\end{aligned}
$$

Соотношения (21)-(27) определяют дрейфовые и циклические компоненты скорости материальных частиц верхней и нижней сред. В таком виде они максимально согласованы с соотношением (10) - законом изменения во времени формы границы раздела сред. Циклические компоненты имеют первый порядок малости по амплитуде волны и описаны только главными линейными по амплитуде слагаемыми своего асимптотического представления. Дрейфовые же компоненты скорости в 
линейном по амплитуде приближении равны нулю. Для их учета в смысле главных членов разложения по амплитуде волны нужно использовать выражения, пропорциональные квадрату амплитуды. Качественно различные элементы динамического поведения - циркуляционное и переносное движения материальной частицы - оказываются совместно учтенными, посредством сохранения в каждом случае своего главного члена асимптотического разложения.

\section{5. Примеры расчета траекторий материальных частиц}

Траектории материальных частиц вычисляются интегрированием по времени циклических и дрейфовых компонент поля скоростей в представлении Лагранжа. Чтобы не загромождать изложение, ограничимся уравнениями формы границы раздела и траекторий движения материальных частиц, непосредственно прилегающих к этой границе, в условиях, когда $U_{0}^{2} \leq U_{\mathrm{cr}}^{2}(k)$ (при этом параметр $\omega$ является действительным числом), т.е. для волнового возмущения, представляющего собой периодическую бегущую волну постоянной амплитуды:

$$
\begin{gathered}
\xi=a \cos (\omega t-k x) \\
X=x_{0}+a\left(\sin \left(k x_{0}\right)+\sin \left(\omega\left(1-a^{2} k^{2}\right) t-k x_{0}\right)\right)+a^{2} k \omega t \\
Z=a \cos \left(\omega\left(1-a^{2} k^{2}\right) t-k x_{0}\right) \\
X^{\prime}=x_{0}^{\prime}+U_{0} t-a\left(\sin \left(k x_{0}^{\prime}\right)+\sin \left(\left(\omega-k U_{0}\right)\right.\right. \\
\left.\left.\times\left(1-a^{2} k^{2}\right) t-k x_{0}^{\prime}\right)\right)+a^{2} k\left(\omega-k U_{0}\right) t \\
Z^{\prime}=a \cos \left(\left(\omega-k U_{0}\right)\left(1-a^{2} k^{2}\right) t-k x_{0}^{\prime}\right)
\end{gathered}
$$

Здесь $X \equiv X\left(x_{0}, z_{0}, t\right)$ и $Z \equiv Z\left(x_{0}, z_{0}, t\right)$ - текущие координаты материальной частички нижней среды, начавшей двигаться из положения $\left(x_{0}, z_{0}\right)$. Текущие координаты частички верхней среды отмечены штрихом. Само положение $\left(x_{0}, z_{0}\right)=\left(x_{0}, \xi\left(x_{0}, 0\right)\right)=\left(\left(x_{0}, a \cos \left(k x_{0}\right)\right)\right)-$ точка на поверхности раздела, к которой частички сред прилегают снизу и сверху при $t=0$. При построении (28)-(32) амплитудные множители циклических слагаемых упрощались до своего главного первого приближения по амплитуде волны. Например:

$$
\begin{aligned}
a \exp \left( \pm k z_{0}\right) & =a \exp \left( \pm k \xi\left(x_{0}, t\right)\right) \\
& =a\left(1 \pm k a \cos \left(\omega t-k x_{0}\right)+\ldots\right) \approx a .
\end{aligned}
$$

В соотношении (28) координата $x$ не снабжена индексом „0“, поскольку этой формулой описывается эволюция формы поверхности, как геометрического места точек пространства.

Несложно заметить, что отношение скорости $V_{S}=$ $=a^{2} k \omega$, с которой дрейфует прилегающая к границе раздела материальная частичка нижней среды (см. последнее слагаемое в (29)), к фазовой скорости волнового движения $V_{\mathrm{ph}}=\omega / k$ равно $a^{2} k^{2}$. За период $T=\lambda / V_{\mathrm{ph}}$ материальная частичка переносится на расстояние $S_{T}=V_{S} T=a^{2} k^{2} \lambda$. Таким образом, справедливы формулы:

$$
\frac{V_{S}}{V_{\mathrm{ph}}}=\frac{S_{T}}{\lambda}=a^{2} k^{2} .
$$

Для того чтобы переместиться на расстояние, равное длине волны $\lambda$, частичке нижней среды требуется время $t_{\lambda}=\lambda / V_{S}=\lambda /\left(V_{\mathrm{ph}} a^{2} k^{2}\right)=T /\left(a^{2} k^{2}\right)$. В долях периода волнового движения имеем

$$
\frac{t_{\lambda}}{T}=\frac{1}{a^{2} k^{2}} .
$$

Несложно получить аналогичные формулы для материальной частички верхней среды, дрейфующей со своей стороны границы раздела:

$$
\begin{gathered}
\frac{V_{S}^{\prime}}{V_{\mathrm{ph}}}=\frac{S_{T}^{\prime}}{\lambda}=\beta+a^{2} k^{2}(1-\beta) ; \\
\frac{t_{\lambda}^{\prime}}{T}=\frac{1}{\beta+a^{2} k^{2}(1-\beta)} ; \quad \beta=\frac{U_{0}}{V_{\mathrm{ph}}} .
\end{gathered}
$$

Рассмотрим примеры расчета движений материальных частиц верхней и нижней сред, прилегающих к искаженной периодическим волновым движением границе раздела. Расчеты выполнялись при значениях параметров задачи, соответствующих границе вода-воздух в нормальных условиях: $\rho=1 \mathrm{~g} / \mathrm{cm}^{3} ; \rho^{\prime}=0.001 \mathrm{~g} / \mathrm{cm}^{3}$; $\gamma=72 \mathrm{dyn} / \mathrm{cm}$. В качестве величины волнового числа использовалось значение:

$$
k=k_{*}=\sqrt{\frac{g\left(\rho-\rho^{\prime}\right)}{\gamma}} \approx 3.69 \mathrm{~cm}^{-1},
$$

при котором правая часть соотношения (15), рассматриваемая как функция от $k$, достигает минимума. Это соответствует длине волны $\lambda_{*}=2 \pi / k_{*} \sin 1.70 \mathrm{~cm}$, наиболее „чувствительной“ к дестабилизирующему влиянию движения верхней среды. Для такого волнового искажения критическое значение скорости верхней среды $U_{\mathrm{cr}}=U_{\mathrm{cr}}\left(k_{*}\right) \sim 730 \mathrm{~cm} / \mathrm{s}$ (см. (15)), по превышении которого амплитуда волны становится растущей во времени, является наименьшим в сравнении с волновыми возмущениями, обладающими другими значениями $k \neq k_{*}$. Амплитуда волны принималась равной полмиллиметра, что составляет примерно 0.03 ее длины. С одной стороны это обеспечивает требуемую в модели существенную малость амплитуды по сравнению с длиной, а с другой - такое значение амплитуды оказывается вполне достаточным для наглядной иллюстрации основных свойств движения приповерхностных материальных частиц обеих сред.

Рис. 1 показывает, как происходит движение поверхности и прилегающих к ней материальных частиц в 

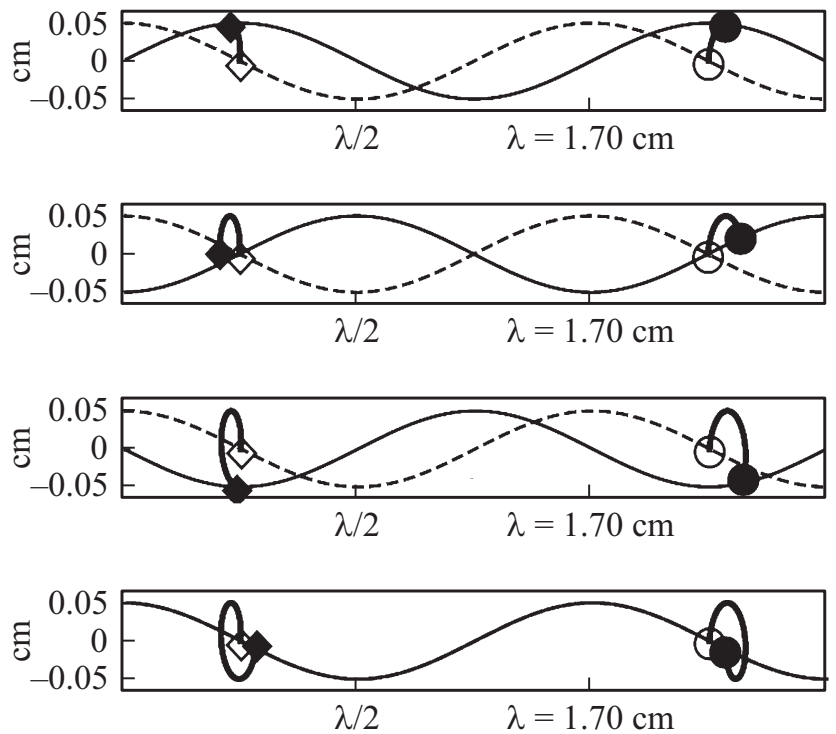

Рис. 1. Положения профиля волны (сплошная линия) и прилегающих к нему материальных частиц, рассчитанные по формулам (28)-(32) в отсутствии сдвигового течения $U_{0}=0 \mathrm{~cm} / \mathrm{s}$; при $\rho=1 \mathrm{~g} / \mathrm{cm}^{3} ; \rho^{\prime}=0.001 \mathrm{~g} / \mathrm{cm}^{3} ; \gamma=72 \mathrm{dyn} / \mathrm{cm}$; $k=3.7 \mathrm{~cm}^{-1}$ в последовательные моменты времени $\mathrm{t}$ - сверху вниз: $t=T / 4 ; T / 2 ; 3 T / 4 ; T(T=2 \pi / \omega-$ период волнового движения); о и $\diamond-$ положения материальных частиц нижней и верхней сред при $t=0$, • и $\downarrow$ их текущие положения; штрихи - профиль волнового искажения при $t=0$.

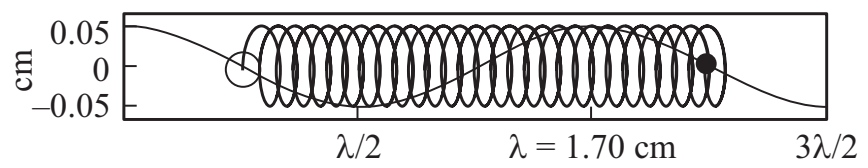

Рис. 2. Траектория частицы нижней среды, описанная за время $t=29 \mathrm{~T}$, рассчитанная по формулам (29), (30) при тех же значения параметров и в тех же обозначениях, что и на рис. 1 .

условиях, когда относительное движение сред отсутствует $\left(U_{0}=0\right)$, а косинусоидальное начальное возмущение границы раздела (штриховой профиль) со временем перемещается вправо (сплошной профиль). Показаны последовательные положения прилегающих к границе материальных частиц верхней и нижней сред с временным шагом $\Delta T=T / 4$ в четверть периода волнового движения $T=2 \pi / \omega$. Незачерненный кружок и ромбик отмечают начальные положения материальных частиц нижней и верхней сред соответственно. Аналогичные зачерненные символы обозначают текущие положения тех же частиц. Видно, что при отсутствии в системе сдвигового течения частички обеих сред совершают противоположно ориентированные петлеобразные движения. Вертикальный „размах“ обоих витков равен удвоенной амплитуде волны. Обе частички с течением времени смещаются в направлении распространения волны с одинаковой средней дрейфовой скоростью (см. последние слагаемые в (29) и (31)).
На рис. 2, построенном с помощью (28)-(30), показана петлеобразная траектория частички нижней среды за 29 периодов волнового движения $T=2 \pi / \omega$. Согласно (33), $t_{\lambda} / T=a^{-2} k^{-2} \sim 29.4-$ время в единицах периода, за которое средний дрейф сносит частицу на длину волны. Если время измерять в долях периода волнового движения, то рис. 2 останется неизменным при увеличении скорости верхней среды вплоть до критического значения $U_{\mathrm{cr}}\left(k_{*}\right) \sim 730 \mathrm{~cm} / \mathrm{s}$.

Рис. 3 построен с помощью соотношений (28), (31), (32) и показывает, что даже незначительное увеличение скорости сдвигового течения до $U_{0}=10 \mathrm{~cm} / \mathrm{s}$ (направлена вправо) существенно изменяет форму траектории движения его материальных частиц. Петлеобразные движения разворачиваются в дугообразные с существенным смещением частицы в направлении общего движения верхней среды. При этом, согласно (34), в условиях, когда $U_{0}<V_{\mathrm{ph}}$, частичка верхней среды за период волнового движения сносится по горизонтали меньше, чем на длину волны, а дополнительный к общему движению со скоростью $U_{0}$ дрейф (см. последнее слагаемое (31)) направлен в ту же сторону, что и общее движение верхней жидкости. Период циклического движения ма-
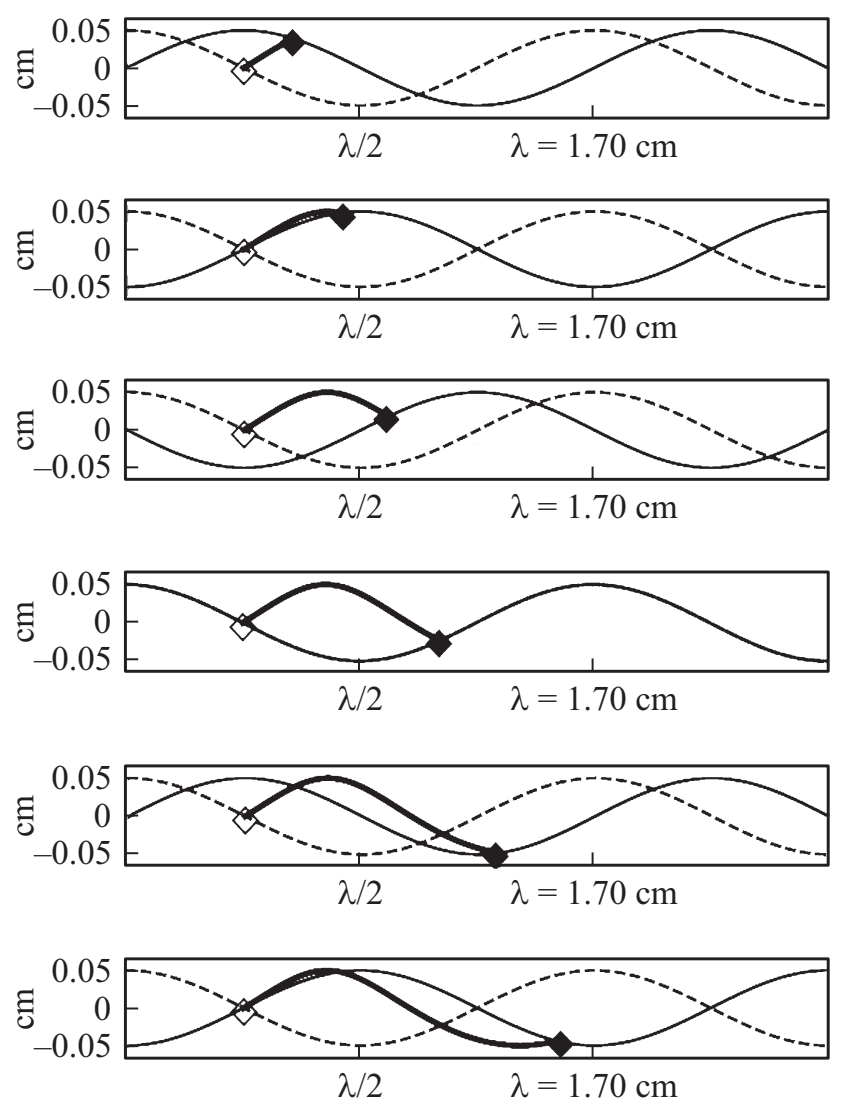

Рис. 3. Положение профиля волны и траектории прилегающей к нему материальной частицы верхней среды, рассчитанные по формулам (28), (31), (32) при $U_{0}=10 \mathrm{~cm} / \mathrm{s}$ в последовательные моменты времени $t-$ сверху вниз: $t=T / 4 ; T / 2 ; 3 T / 4$; $T ; 5 T / 4 ; 3 T / 2$. Значения остальных параметров и обозначения такие же, как для рис. 1. 

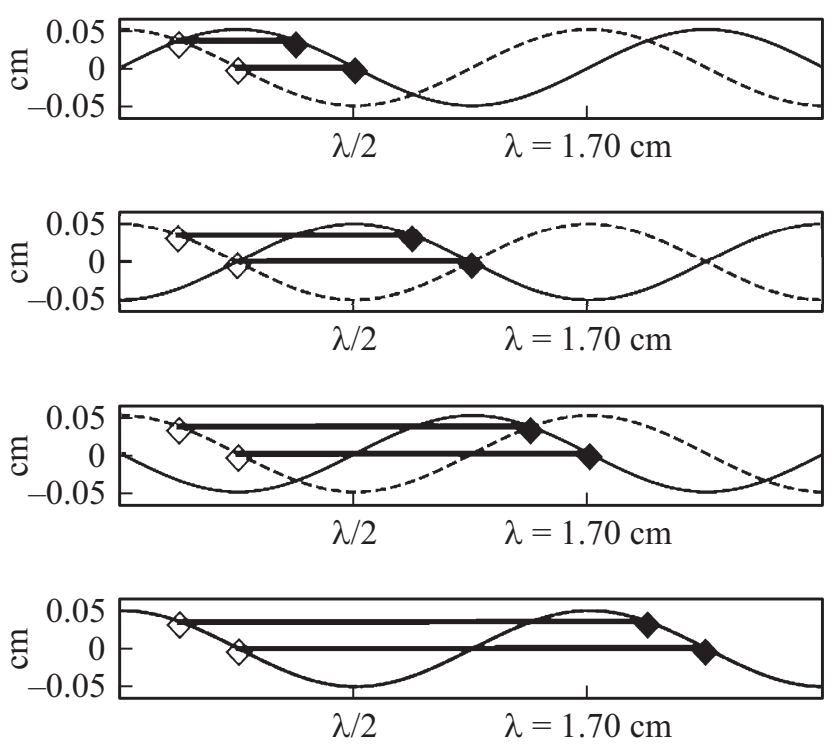

Рис. 4. Положение профиля волны и траектории прилегающей к нему материальной частицы верхней среды, рассчитанные по формулам (28), (31), (32) при $U_{0}=23 \mathrm{~cm} / \mathrm{s}$ в последовательные моменты времени $t-$ сверху вниз: $t=T / 4 ; T / 2 ; 3 T / 4 ; T$. Значения остальных параметров и обозначения такие же, как для рис. 1.

териальной частицы (измеряемый, например, временем между двумя ее последовательными верхними положениями) при $U_{0}<V_{\mathrm{ph}}$ оказывается больше периода волнового движения $T$. С дальнейшим ростом $U_{0}$ дрейфовая добавка, описываемая последним слагаемым (31), в верхней среде уменьшается и при $U_{0}=V_{\mathrm{ph}}=\omega / k$ обнуляется, а период циклического движения частицы верхней среды $T /\left(\omega-k U_{0}\right)$ растет до бесконечности. При $U_{0}=V_{\mathrm{ph}}$ частицы верхней среды двигаются равномерно и прямолинейно (рис. 4) с фазовой скоростью волны $V_{\mathrm{ph}} \sim 23 \mathrm{~cm} / \mathrm{s}$.

Рис. 5 иллюстрирует, как перемещается прилегающая к возмущенной волновым движением границе сред частичка верхней области течения в условиях, когда $V_{\mathrm{ph}}<U_{0}<U_{\mathrm{cr}}\left(k_{*}\right)\left(U_{0}=50 \mathrm{~cm} / \mathrm{s}\right)$. Видно, что частичка верхней среды двигается по сильно растянутой вдоль горизонтали дугообразной траектории. За период волнового движения она проходит вдоль $O x$ расстояние, превышающее длину волны. При этом дрейфовая добавка (последнее слагаемое в (31)) в верхней среде оказывается направленной против течения $U_{0}$, и чем больше величина $U_{0}$, тем больше становится скорость этой составляющей.

Если $U_{0}>U_{\text {cr }}$, то формулы (29)-(32) не справедливы. Теперь при интегрировании поля скоростей следует учитывать комплексность частоты $\omega$, и, значит, экспоненциальный расчет амплитуды волнового возмущения с течением времени. Система быстро выходит за пределы применимости модели, подразумевающей малость амплитуды по сравнению с длиной волны. И все же, формулы (22) и (25) позволяют проследить тенденции поведения дрейфового движения на начальной стадии развития неустойчивости. Используя выражение для $\sigma$ (см. (23)), несложно убедиться, что (22) и второе слагаемое формулы (25) имеют противоположные знаки. Это означает, что начальная стадия развития неустойчивости Кельвина-Гельмгольца проявляется не только в росте амплитуды волнового искажения границы раздела, но и в возникновении в обеих средах экспоненциально нарастающих со временем встречных по отношению друг к другу дрейфовых течений. В нижней среде нарастает дрейфовое течение (21), направленное вдоль направления сноса верхней среды, в которой в свою очередь перенос со скоростью $U_{0}$ ослабляется вторым слагаемым (25) - „встречным противодрейфом“. Получается, что в начале развития неустойчивости нижнее и верхнее дрейфовые течения эволюционируют таким образом, чтобы с течением времени уменьшить величину тангенциального скачка относительной скорости сред на их границе. Таким образом, уже на начальном этапе развития неустойчивости запускается механизм ее ослабления - уменьшения величины дестабилизирующего границу тангенциального скачка скорости. При этом искажение границы раздела растет со временем пропорционально $\sim a \exp (r t)$, а скорость встречных дрейфовых потоков, стремящихся сократить тангенциальный скачок скорости, нарастет быстрее: $\sim a^{2} \exp (2 r t)$. Качественно сказанное можно интерпретировать как наличие в рассматриваемой динамической системе отрицательной обратной связи, препятствующей неограниченному нарастанию неустойчивости.
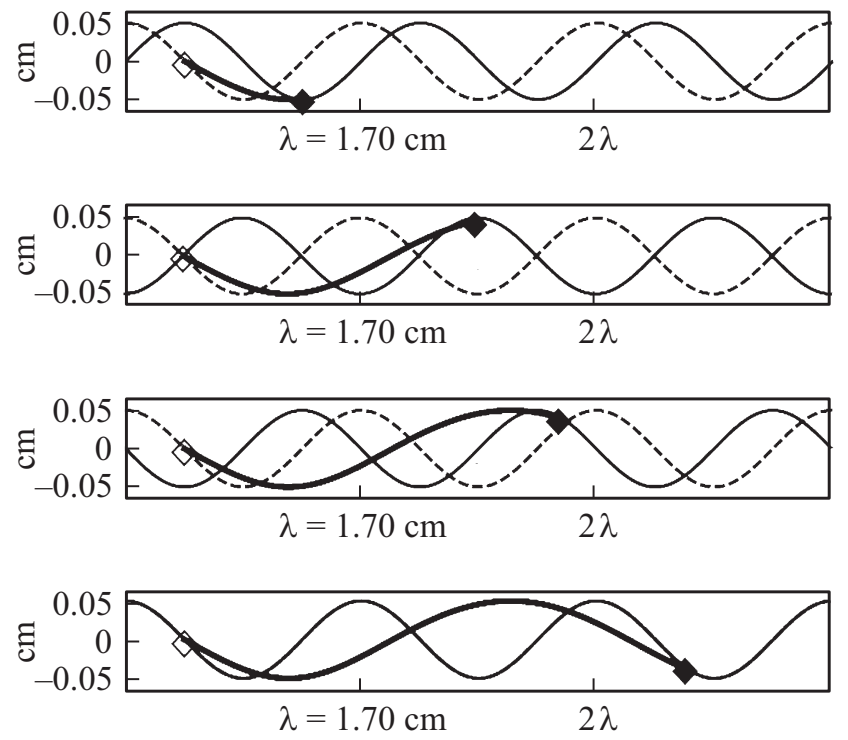

Pис. 5. Положение профиля волны и траектории прилегающей к нему материальной частицы верхней среды, рассчитанные по формулам (28), (31), (32) при $U_{0}=50 \mathrm{~cm} / \mathrm{s}$ в последовательные моменты времени $t$ - сверху вниз: $t=T / 4 ; T / 2 ; 3 T / 4 ; T$. Значения остальных параметров и обозначения такие же, как для рис. 1. 


\section{Заключение}

В настоящей работе предложена аналитическая асимптотическая методика расчета циклических и дрейфовых движений материальных частиц, находящихся по разные стороны от искаженной периодическим волновым возмущением границы раздела двух сред, участвующих в относительном сдвиговом смещении. Основные приемы расчета продемонстрированы в рамках модели идеальных жидкостей, ограниченных лишь поверхностью раздела, но концептуально применимы и в более сложных ситуациях. Установлены правила преобразования аналитических выражений для эйлерова поля скоростей, позволяющие перейти к аналитическому описанию движения отдельных материальных и в некоторых случаях установить новые свойства хорошо известного течения. В частности, обнаружилось новое свойство классической неустойчивости Кельвина-Гельмгольца: она, как оказалось, проявляется не только в нарастании амплитуды искажения границы раздела двух смещающихся относительно друг друга сред, но и в появлении по разные стороны от этой границы усиливающихся со временем встречных приповерхностных дрейфовых течений, стремящихся сократить величину тангенциального скачка скорости, спровоцировавшего неустойчивость.

\section{Список литературы}

[1] Stoks G.G. // Cambridge Phil. Soc. 1847. Vol. 8. N 4. P. 441455.

[2] Ландау Л.Д., Лифиии, Е.M. Теоретическая физика: Учебное пособие. В 10 т. Т. VI. Гидродинамика. 3-е изд. М.: Наука, 1986. 736 с.

[3] Левич В.Г. Физико-химическая гидродинамика. 2-е изд. М.: ГИФМЛ, 1959. 699 с

[4] Сивухин Д.В. Общий курс физики. Т. II. Термодинамика и молекулярная физика. М.: Наука, 1975. 552 с.

[5] Clamond D. // J. Fluid Mech. 2007. Vol. 589. P. 433-454.

[6] Longuent-Higgens M.S. // Roy. Soc. Lond. Tr. Ser. A. 1953. Vol. 245. N 903. P. 535-581.

[7] Longuent-Higgens M.S. // J. Fluid. Mech. 1986. Vol. 173. P. 683-707.

[8] Weber J.E. // J. Phys. Oceanogr. 1983. Vol. 13. P. 524-530.

[9] Weber J.E. // J. Fluid. Mech. 1983. Vol. 137. P. 115-129.

[10] Craik A.D.D. // J. Fluid. Mech. 1982. Vol. 116. P. 187-205.

[11] Степаняни, Ю.А., Фабрикант А. // УФН. 1989. Т. 159. Вып. 1. C. 83-123.

[12] Siddiqui M.H.K., Loewen M.R. // J. Fluid. Mech. 2007. Vol. 573. P. 417-456.

[13] Абрашкин А.А., Якубович Е.И. Вихревая динамика в лагранжевом описании. М.: Физматлит, 2006. 176 с.

[14] Белоножкко Д.Ф., Козин А.В. // Изв. РАН. МЖГ. 2011. № 2. C. $112-120$

[15] Бэтчелор Джк.К. Введение в динамику жидкости. Москва-Ижевск: НИЦ „Регулярная и хаотическая динамика“, 2004. $768 \mathrm{c.}$

[16] Фабер Т.Е. Гидроаэродинамика. М.: Постмаркет, 2001. $560 \mathrm{c}$.
[17] Дразин $\Phi$. Введение в теорию гидродинамической устойчивости. М.: Физматлит, 2005. 288 с. Drazin P.G. Introduction to Hydrodynamic Stability. Cambridge (University Press., 2002. 278 p.).

[18] Ле Блон П., Майсек Л. Волны в океане. Ч. 1. М.: Мир, $1981.480 \mathrm{c}$.

[19] Белоножко Д.Ф., Очиров А.А. // ЭОМ. 2016. Т. 52. № 1. C. 91-97.

[20] Christensen K.H., Terrile E. // J. Fluid Mech. 2009. Vol. 620. P. 313-332.

[21] Phillips O.M. The Dynamics of the Upper Ocean. Cambridge University Press., 1977. 336 p. 